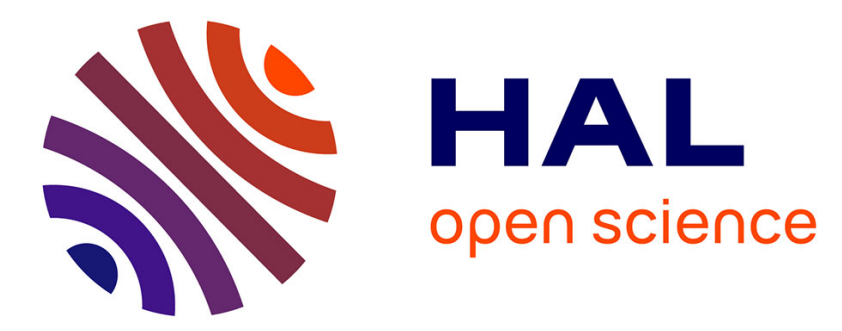

\title{
Laser action along and near the optic axis of a holmium-doped KY(WO 4 ) 2 crystal
}

Romain Cattoor, Inka Manek-Hönninger, Daniel Rytz, Lionel Canioni, Marc Eichhorn

\section{- To cite this version:}

Romain Cattoor, Inka Manek-Hönninger, Daniel Rytz, Lionel Canioni, Marc Eichhorn. Laser action along and near the optic axis of a holmium-doped KY(WO 4 ) 2 crystal. Optics Letters, 2014, 39 (22), pp.6407-6410. 10.1364/OL.39.006407 . hal-01094397

\section{HAL Id: hal-01094397 https://hal.science/hal-01094397}

Submitted on 12 Dec 2014

HAL is a multi-disciplinary open access archive for the deposit and dissemination of scientific research documents, whether they are published or not. The documents may come from teaching and research institutions in France or abroad, or from public or private research centers.
L'archive ouverte pluridisciplinaire HAL, est destinée au dépôt et à la diffusion de documents scientifiques de niveau recherche, publiés ou non, émanant des établissements d'enseignement et de recherche français ou étrangers, des laboratoires publics ou privés. 


\title{
Laser action along and near the optic axis of a holmium-doped $\mathrm{KY}\left(\mathrm{WO}_{4}\right)_{2}$ crystal
}

\author{
Romain Cattoor,,${ }^{1,2, *}$ Inka Manek-Hönninger, ${ }^{1}$ Daniel Rytz, ${ }^{3}$ Lionel Canioni, ${ }^{1}$ and Marc Eichhorn ${ }^{2}$ \\ ${ }^{1}$ Laboratoire Ondes et Matière d'Aquitaine (LOMA-CNRS-UMR5798, University of Bordeaux), \\ 351 Cours de la Libération, 33405 Talence, France \\ ${ }^{2}$ Institut Franco-Allemand de Recherches de Saint-Louis (ISL), 5 Rue du Général Cassagnou, 68300 Saint-Louis, France \\ ${ }^{3}$ FEE GmbH Struthstr. 2, 55743 Idar-Oberstein, Germany \\ *Corresponding author: romain.cattoor@isl.eu
}

Received August 14, 2014; revised September 29, 2014; accepted October 10, 2014; posted October 14, 2014 (Doc. ID 216844); published November 5, 2014

\begin{abstract}
We demonstrate the first (to our knowledge) quasi-three-level conical refraction laser operating at $2 \mu \mathrm{m}$, with $1.6 \mathrm{~W}$ of output power at $2074 \mathrm{~nm}$, using a holmium-doped $\mathrm{KY}\left(\mathrm{WO}_{4}\right)_{2}$ crystal. A maximum slope efficiency of $52 \%$ has been achieved, along the optic axis with respect to the absorbed pump power. Furthermore, lasing operation around the optic axis has been performed. In this case, a maximum output power of $3 \mathrm{~W}$ has been reached, with a slope efficiency better than 70\%, which are the best performances ever reported on this material. (c) 2014 Optical Society of America OCIS codes: (140.0140) Lasers and laser optics; (140.3300) Laser beam shaping; (140.3410) Laser resonators; (140.3580) Lasers, solid-state.

http://dx.doi.org/10.1364/OL.39.006407
\end{abstract}

Studies of conical refraction (CR) have recently revived. When a Gaussian light beam propagates along the optic axis of a biaxial crystal, under certain conditions a two ring-shaped beam can be observed, at the exit face of the crystal. Several publications describe this effect, theoretically and experimentally [1-4]. Recent availability of long biaxial crystals, with good optical quality, facilitates CR studies. Therefore, several applications of this effect have recently been investigated, such as optical tweezers []], particle trapping []], beam shaping [ㅁ-11], and microscopy [12]. There are also some publications about laser operation along an optic axis of a biaxial crystal, the first publication being [13]. Using a short (3 mm long) biaxial crystal of a $\overline{\mathrm{CR}}$-cut ytterbium-doped $\mathrm{KGd}\left(\mathrm{WO}_{4}\right)_{2}(\mathrm{KGW})$, the output polarization of their laser was modified, by translating the output coupler mirror. The researchers obtained a slope efficiency of $60 \%$ along the $\mathrm{CR}$ axis, which is similar to the value obtained for a classical $\mathrm{N}_{g}$-cut orientation. However, the threshold was $2 \times$ higher for CR orientation. A few years later, $[14,15]$ presented a four-level conical refraction laser, made with neodymium-doped KGW. The researchers obtained both a Gaussian and CR output beam. They reported a slope efficiency close to the quantum defect limit [14], and no influence of any thermal effect over the measurement range (up to $3 \mathrm{~W}$ at $1067 \mathrm{~nm}$ ) was observed. The thermo-optic properties of neodymium-doped KGW were studied in [16]. The researchers show the anisotropy of the thermo-optic properties, along this propagation direction with a short crystal (1 mm long). However, the effect of CR inside the cavity, changing the light distribution inside the crystal, is still not well-known.

In this Letter, we report the first experimental results (to our knowledge) of a holmium-doped $\mathrm{KY}\left(\mathrm{WO}_{4}\right)_{2}$ (KYW) CR laser, showing laser action along the $\mathrm{CR}$ axis. Measurements near the optic axis have also been performed, in order to be able to compare CR and Gaussian beam intracavity laser performance.

For all measurements, we used the same setup. Only the crystal was tilted. We used a home-made thulium fiber laser as a pump source, emitting at $1.96 \mu \mathrm{m}$, with a linewidth below $0.2 \mathrm{~nm}$, a maximum output power around $6 \mathrm{~W}$, and a Glan-polarizer in order to obtain a linearly polarized pump beam, with a half-wave plate to rotate the polarization. The beam was focused to a waist of $100 \mu \mathrm{m}$. The first power meter (Fig. 1) catches a few percent of the pump laser passing through the mirror at $45^{\circ}$, and was calibrated to provide the incident pump power on the crystal. The laser cavity was composed of one dichroic mirror, which transmitted at $1.96 \mu \mathrm{m}$ and was highly reflective above $2.02 \mu \mathrm{m}$, with a $91 \mathrm{~mm}$ radius of curvature. The output coupler had $95 \%$ reflectivity at $1.96 \mu \mathrm{m}$, and $95.5 \%$ at $2.06 \mu \mathrm{m}$, with a $300 \mathrm{~mm}$ radius of curvature. We pumped longitudinally, and the pump wavelength was retro-reflected into the crystal. The $12 \mathrm{~mm}$ long holmium-doped KYW crystal (1\% at.), provided by FEE GmbH, was close to the output coupler $(\sim 4 \mathrm{~mm})$ and mounted into a water-cooled heat sink, which was held around $20^{\circ} \mathrm{C}$, with the $\mathrm{N}_{m}$ axis parallel to the optical table. It has an anti-reflective coating for the pump and lasing wavelength. The cutting angle of the crystal was $18^{\circ}$ between the crystallographic $c$-axis and the dielectric axis $\mathrm{N}_{g}$. The cutting angle between the optic axis and the $\mathrm{N}_{g}$ axis was $44^{\circ}$, which is closed to the values given in [17]. However, the misalignment with

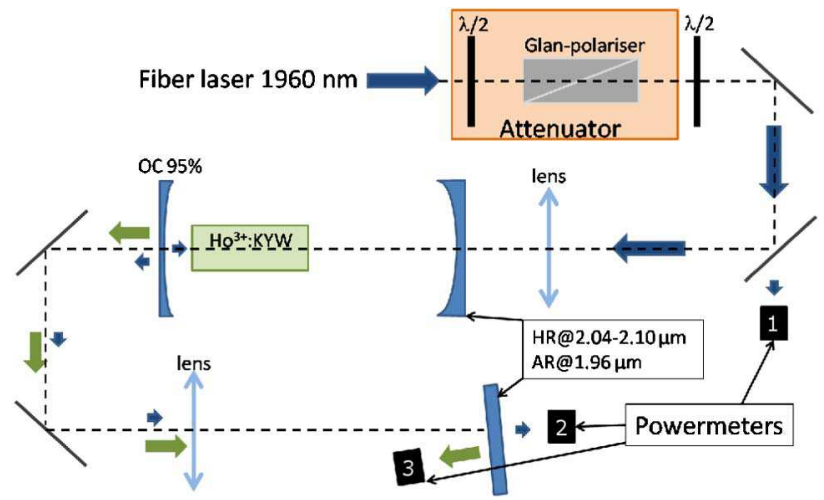

Fig. 1. Laser setup. 
respect to the optic axis at $2 \mu \mathrm{m}$ is close to $\sim 1^{\circ}$ using those angles. The semi-angle of the $\mathrm{CR}$ cone for such a crystal is $\sim 20 \mathrm{mrad}$, and the ring radius for a crystal length of $12 \mathrm{~mm}$ is $240 \mu \mathrm{m}$. The total cavity length was about $65 \mathrm{~mm}$. The minimum cavity mode size has a radius of $170 \mu \mathrm{m}$, which is bigger than the pump waist, and therefore not optimized for mode matching. However, as we apply a double-pass of the pump, which diverges during propagation, experimentally we found out that this mode size was the optimum compromise in this cavity configuration. The second pass had a beam radius of $200 \mu \mathrm{m}$ at the exit face. Those mode sizes are calculated without taking into account any thermal lens.

Figure 2 shows the raw measurements of the output power versus the incident pump power, for crystal orientations along the optic axis (CR-axis), and tilted away $\left(>1^{\circ}\right)$ from the optic axis (off-CR-axis). For the latter, two pump polarizations were investigated $\left(\mathrm{E} \perp \mathrm{N}_{m}\right.$ and $\mathrm{E} \| \mathrm{N}_{m}$, with $\mathrm{N}_{p}, \mathrm{~N}_{m}$, and $\mathrm{N}_{g}$ the optical indicatrix axes considering $n_{p}<n_{m}<n_{g}$ ). The nonlasing absorption of the crystal at $1960 \mathrm{~nm}$ was measured to be 1.5 and $0.4 \mathrm{~cm}^{-1}$, for $\mathrm{E} \| \mathrm{N}_{m}$ and $\mathrm{E} \perp \mathrm{N}_{m}$, respectively. The best efficiency is obtained for an off-CR-axis alignment with the $\mathrm{E} \| \mathrm{N}_{m}$ polarized pump beam, and the worst efficiency with $\mathrm{E} \perp \mathrm{N}_{m}$. In the case of the CR laser, the threshold and the efficiency with the $\mathrm{E} \| \mathrm{N}_{m}$ polarized pump beam is in-between the other off-CR-axis results. With such curves, one might conclude that the $\mathrm{CR}$ laser behavior is mainly driven by the spectroscopic properties along the optic axis. However, this is not the case.

The laser was first aligned off-CR-axis ( $1^{\circ}$ away). Once the mode-matching between the cavity mode and the pump beam was optimized at full power, the crystal was tilted in order to obtain CR lasing. When the crystal orientation becomes closer to the optic axis, the laser power drops down to $0 \mathrm{~W}$. Then, when the optic axis is reached, a few hundred $\mathrm{mW}$ can be measured. The output power is optimized as the beam profile, by slightly moving the pump beam and mirrors.

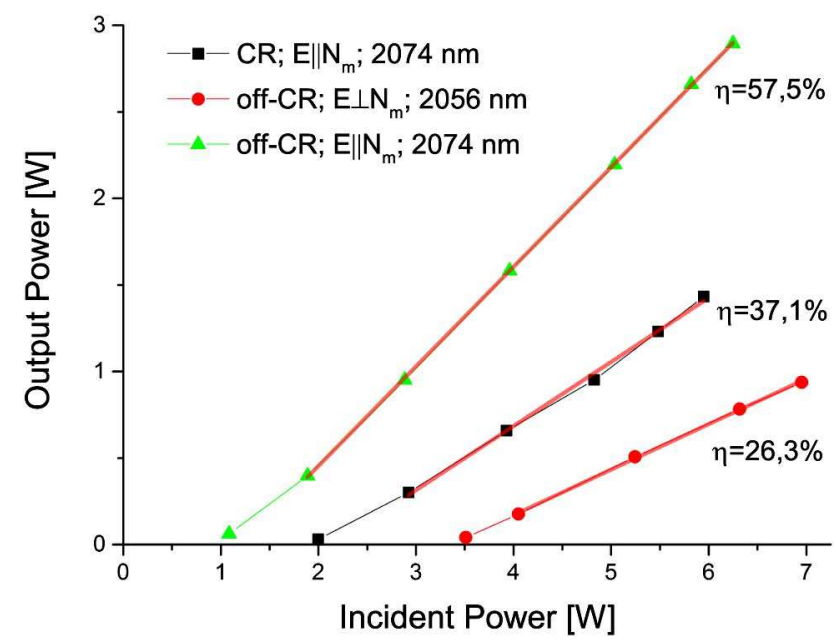

Fig. 2. Output power versus incident pump power, for CR and off-CR-axis. Red curves are the linear fits giving the slope efficiency. Legend lists orientation, pump polarization, and lasing wavelength.
Figure 3 depicts the output power obtained over the absorbed pump power, for CR and non-CR crystal alignment.

The total absorbed pump power is estimated by measuring the $5 \%$ of the pump passing through the output coupler, assuming that the absorption for the first and second pass is the same. In fact, at the output of the laser, a dichroic mirror has been used in order to measure separately the lasing wavelength power, and the rest of the pump power, with two different powermeters. With such a configuration, we were able to measure simultaneously the incident pump power, the output power, and the rest of the pump power. With this last measurement, it is possible to deduce the absorbed pump power. The measurement given by the powermeter is the fraction of the power passing through the output coupler, $P_{m}=T_{\mathrm{OC}} P_{1}$, with $P_{1}$ the power left after the first pass. The linear absorption can be deduced using $P_{1}=P_{i} e^{-\alpha l}$. The absorbed pump power for the first pass is $P_{\text {abs } 1}=P_{i}-P_{1}$, and for the second pass we assume that the linear absorption is the same, $P_{\text {left }}=R_{\mathrm{OC}} P_{1} e^{-\alpha l}$. This gives $P_{\text {abs } 2}=R_{\mathrm{OC}} P_{1}-P_{\text {left }}$. The total absorbed pump power is $P_{\text {abs }}^{\text {tot }}=P_{\text {abs1 }}+P_{\text {abs2 } 2}$.

For the CR orientation the threshold is around $1.3 \mathrm{~W}$, with a slope efficiency of $52 \%$, with respect to the absorbed pump power. A maximum slope efficiency of $72 \%$ has been achieved for the orientation off-CR-axis. The percentage of the absorbed pump power at the maximal output power is estimated to be 76,29 , and $67 \%$, for $\mathrm{E} \| \mathrm{N}_{m}$ off-CR-axis, $\mathrm{E} \perp \mathrm{N}_{m}$ off-CR-axis, and the CR-laser, respectively.

Along the $\mathrm{CR}$ orientation, the threshold is $2 \times$ higher than in the off-CR-axis case. This has also been observed in [13], but can not be only explained by the difference of the absorption cross section along this direction, since both curves of off-CR-axis lasers are similar. In our case, when the pump waist is small enough to observe the CR ring, the pump fluence might decrease due to the $\mathrm{CR}$

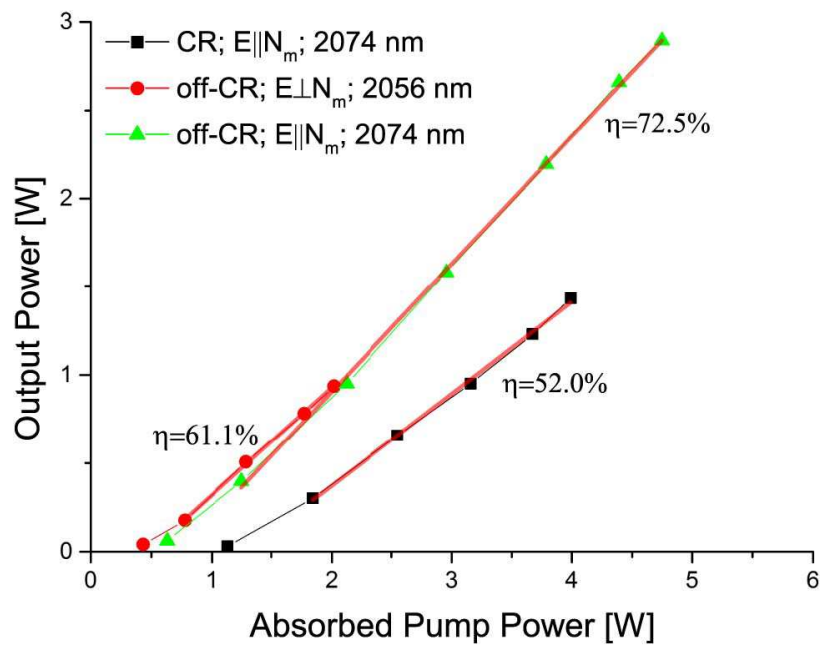

Fig. 3. Output power versus absorbed pump power, for CR and off-CR axis. Red curves are the linear fits giving the slope efficiency. Legend lists orientation, pump polarization, and lasing wavelength. 


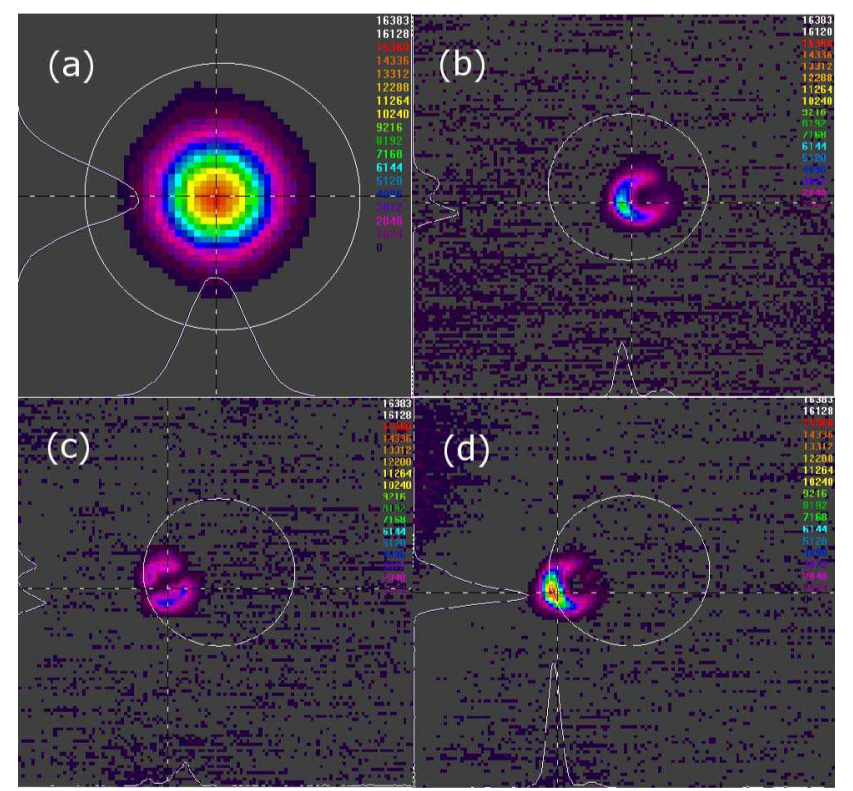

Fig. 4. Beam profile at full power; (a) far field off-CR-axis; (b) near field along CR; (c) near field along CR, with analyzer $\mathrm{E} \perp \mathrm{N}_{m}+60^{\circ}$; and (d) near field along $\mathrm{CR}$, with analyzer $\mathrm{E} \perp \mathrm{N}_{m}-30^{\circ}$.

pattern. This could explain why the laser threshold is higher for the CR laser.

We obtain the conical refraction output behind the output coupler, which has a lower radius of curvature compared to the other mirror. In [15], a Gaussian-like output is reported after a flat output coupler. This difference is linked to the laser behavior, as we have to consider the differences between four-level or quasi-three-level laser systems. In the first case, it is the cavity that will control the CR output. In the second case, due to the re-absorption losses, the CR output is driven by the gain inside the laser medium, which is directly linked to the pump injection. This can be the reason why we obtain clearly inferior efficiency along the $\mathrm{CR}$ orientation. The overlaps between cavity mode and gain might not be optimal in our case. This slight misalignment can also explain the tilt of the polarization of the output CR pattern, which does not correspond to the maximum absorption polarization $\mathrm{E} \| \mathrm{N}_{m}$.

In this Letter, we show the best slope efficiency and the maximal output power ever reported in holmiumdoped KYW, as far as we know. In fact, [18] reported a slope efficiency of almost 59\%, with a maximum of $438 \mathrm{~mW}$, for a pumping wavelength at $1941 \mathrm{~nm}$ in the same material, cut along the $\mathrm{N}_{g}$ axis. In [19] the researchers report a maximal output power of $648 \mathrm{~mW}$ and slope efficiency of 55\%, for a pumping wavelength of $1946 \mathrm{~nm}$ (for a holmium-doped KLuW crystal cut along $\mathrm{N}_{g}$ ).

The $\mathrm{M}^{2}$ has been measured for the orientation off-CRaxis at full power. The beam was circular (Fig. 4), and the $\mathrm{M}^{2}$ value was around 1.0 (Fig. 5). As we obtain a conical refracted output beam for $\mathrm{CR}$ orientation, the $\mathrm{M}^{2}$ can not be measured by simply performed beam waist measurements. Fig. $\underline{4}$ shows the beam profile obtained for both orientations. Two polarizations $\left(\mathrm{E} \perp \mathrm{N}_{m}+60^{\circ}\right.$ and $\mathrm{E} \perp \mathrm{N}_{m}-30^{\circ}$ ) were present in our CR laser, aligned for

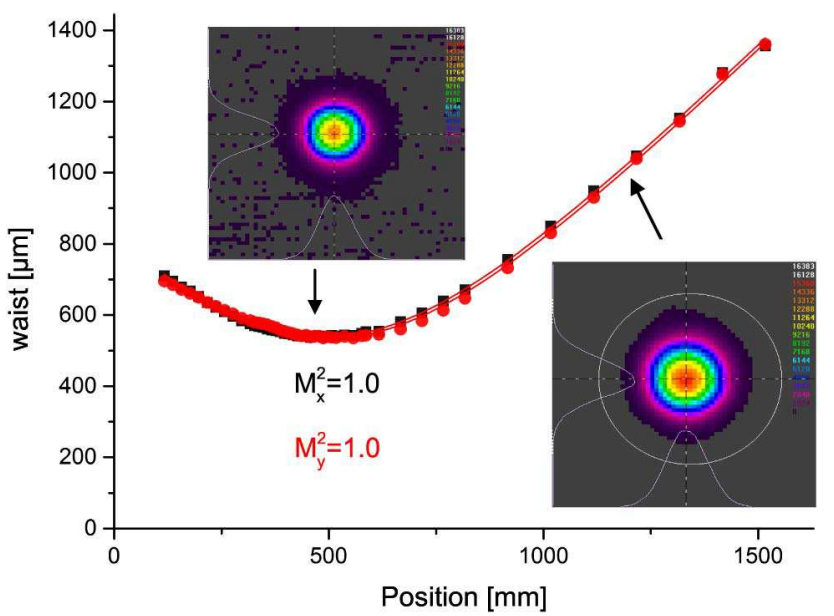

Fig. 5. $\mathrm{M}^{2}$ measurements; the red curves yield an $\mathrm{M}^{2}$ value of 1.0 .

maximal efficiency. However, by slightly moving the end cavity mirror, it is possible to obtain all polarization states as in [13]. However, in that case, the maximal output power drops significantly, depending on the polarization orientation. This can be explained by the fact that we used a polarized pump beam, and so only half of the cone of the CR mode is effectively pumped.

We demonstrated the first (to our knowledge) quasithree-level holmium-doped KYW CR laser with CR output, with a slope efficiency of $52 \%$, related to the absorbed pump power. The maximum output power was $1.6 \mathrm{~W}$. Moreover, near the CR orientation we were able to reach a maximum slope efficiency of $73 \%$, and $3 \mathrm{~W}$ of output power. The difference between the CR and Gaussian laser is essentially due to the overlap between the CR cavity modes, and the gain inside the laser medium.

\section{References}

1. M. V. Berry and M. R. Jeffrey, J. Opt. A 6, 289 (2004).

2. M. V. Berry, J. Opt. A 12, 6651 (2010).

3. A. Turpin, Y. V. Loiko, T. K. Kalkandjiev, and J. Mompart, Opt. Lett. 38, 1455 (2013).

4. G. S. Sokolovskii, D. J. Carnegie, T. K. Kalkandjiev, and E. U. Rafailov, Opt. Express 21, 11125 (2013).

5. D. P. O'Dwyer, K. E. Phelan, C. F. Ballantine, P. R. Rakovich, J. G. Lunney, and J. F. Donegan, Opt. Express 18, 27319 (2010).

6. A. Turpin, V. Shvedov, C. Hnatovsky, Y. V. Loiko, J. Mompart, and W. Krolikowski, Opt. Express 21, 26335 (2013).

7. V. Peet, J. Opt. 12, 095706 (2010).

8. V. Peet, Opt. Lett. 36, 2913 (2011).

9. D. P. O'Dwyer, C. F. Phelan, Y. P. Ralovich, P. R. Eastham, J. G. Lunney, and J. F. Donegan, Opt. Express 19, 2580 (2011).

10. C. F. Phelan, J. F. Donegan, and J. G. Lunney, Opt. Express 19, 21793 (2011).

11. C. F. Phelan, D. P. O'dwyer, Y. P. Rakovich, J. F. Donegan, and J. G. Lunney, Opt. Express 17, 12899 (2009).

12. S. Rosen, G. Y. Sirat, H. Ilan, and A. J. Agranat, Opt. Express 21, 10133 (2013).

13. J. Hellström, H. Henricsson, V. Pasiskevicius, U. Bünting, and D. Haussmann, Opt. Lett. 32, 2783 (2007).

14. A. Abdolvand, K. G. Wilcox, T. K. Kalkandjiev, and E. U. Rafailov, Opt. Express 18, 2753 (2010). 
15. K. G. Wilcox, A. Abdolvand, T. K. Kalkandjiev, and E. U. Rafailov, Appl. Phys. B 99, 619 (2010).

16. P. A. Loiko, V. G. Savitski, A. Kemp, A. A. Pavlyuk, N. V. Kuleshov, and K. V. Yumashev, Laser Phys. Lett. 11, 055002 (2014).

17. P. A. Loiko, V. V. Filippov, N. V. Kuleshov, A. A. Pavlyuk, and K. V. Yumashev, Opt. Commun. 326, 144 (2014).
18. V. Jambunathan, X. Mateos, M. C. Pujol, J. J. Carvajal, M. Aguiló, F. Díaz, U. Griebner, and V. Petrov, Appl. Phys. Express 4, 072601 (2011).

19. X. Mateos, V. Jambunathan, M. C. Pujol, J. J. Carvajal, F. Díaz, M. Aguilól, U. Griebner, and V. Petrov, Opt. Express 18, 20793 (2010). 